\title{
La unidad de análisis como herramienta metodológica de la práctica Psico-Educativa en la escuela
}

\section{The unit of analysis as a methodological tool of psycho-educational practice in the school}

\author{
Camilo Medina Fonseca \\ Universidad Surcolombiana, Colombia \\ camilomedinafonseca@gmail.com
}

\section{Introducción}

El presente artículo es producto de algunas discusiones dadas en la cátedra de psicología educacional de la Universidad de Buenos Aíres Argentina durante el primer cuatrimestre académico de 2016.

La finalidad de este escrito es presentar la propuesta del Circulo Bio-Geo-Gráfico el cual se fundamenta principalmente en el concepto de Unidad de análisis de Vygotsky y que posteriormente Erausquin y $\mathrm{D}^{\prime}$ Arcangelo desarrollan como herramienta de lectura de las situaciones problemáticas emergentes en las instituciones educativas. En este sentido el círculo se muestra como propuesta de 1) unidad de análisis, de 2) cartografía o herramienta de lectura que guíe la unidad de análisis y 3) como parte de los legajos escolares.

\section{Unidad(es) de análisis}

En el capítulo 1 de pensamiento y lenguaje Lev Vygotsky (1995) hace una aproximación al concepto de unidad de análisis en donde lo propone como una segmentación más no como una descomposición, es decir, segmentar en unidades vs descomponer en elementos el objeto a analizar.

En este sentido se busca que la unidad, es decir, la segmentación del conjunto en unidades no pierda las características del conjunto y que a su vez se reconozca como una parte viva e indivisible de la totalidad ya que el análisis de la unidad va a tener como finalidad la construcción de una herramienta de resolución de las cuestiones concretas que se plantean durante la comprensión de un escenario educativo.

Baquero (2012) recapitula la propuesta Vygotskyana afirmando que para aprehender y explicar el objeto o situación de análisis se

"obliga a un ineludible recorte teórico de estos que capture la unidad "viva" de los procesos a explicar... debe adoptarse en principio un modelo de explicación por unidades y no una mera búsqueda de correlaciones o influencias entre elementos o factores que indican sobre un problema". (Pág. 59)

Cabe mencionar que inicialmente el concepto de unidad de análisis en Vygotsky buscaba dar cuenta de cómo estudiar los procesos psicológicos ya que una de las características de ellos es la relación recíproca y la presencia de uno en las tareas del otro borrando cualquier linealidad. Esto va a llevar a autores como Rogoff (como se citó en Baquero, 2002) a trasladar la visión de la unidad de análisis a diferentes cuestiones, principalmente las que impliquen lo individual y lo social. En este sentido "la variación profunda de las unidades de análisis... implica entender lo social y lo individual como co-presentes y mutuamente constitutivos". (Baquero, 2002, pág. 12).

Entre tanto Erausquin y D'Arcangelo (2013) plantean una mirada de la unidad de análisis en pro de la construcción de conocimientos e intervenciones en escenarios educativos retomando la idea expuesta por Rodrigo (Como se citó en Erausquin y D'Arcangelo, 2013) de que el abordaje de un fenómeno concerniente al campo educativo, es decir, 
el plan de intervención a partir del recorte de la problemática en cuestión es construido por los actores a partir de sus modelos mentales. Este aspecto cobra relevancia si se tiene en cuenta que dicha unidad de análisis recortada por el actor es la que -como se ha dicho anteriormente- guiará la intervención de la situación "problema" haciendo foco en uno de los aspectos y así dejando fuera de consideración los demás factores que puedan estar implicados en la situación. Esta visión se contrapone a lo que Baquero (2000) desde una perspectiva contextualista plantea como el ver más allá del fenómeno recortado.

Siguiendo la idea del ver más allá del fenómeno recortado es preciso apoyarnos en la metáfora del zoom de una cámara que al alejar el recorte nos permitirá observar otros elementos del contexto y a su vez el observador se verá invitado “... a re-construir la situación, incluyendo aquellos datos que antes no tenía, y que le permiten ahora representarse la situación de manera más completa y acabada. De la misma manera, el "zoom" que el psicólogo haga al construir la situaciónproblema, la unidad de análisis que recorte, le permitirá abordar la situación de maneras muy diferentes". (Erausquin \& D'Arcangelo. 2013, pág 2).

Este panorama desplegado hasta ahora sobre la unidad de análisis nos lleva de vuelta al origen del concepto Vygotskyano para retomar la idea de que el secreto de la unidad de análisis está en no caer en miradas de fusión o identificación como tampoco en miradas de disyunción y segregación sino que dicha mirada -0 recorteexprese las propiedades del conjunto.

Por lo tanto "La unidad de análisis... hará referencia al recorte que permitirá abordar determinado fenómeno, recorte de la realidad que sea lo suficientemente abarcativo para no vulnerar las características del mismo, pero a su vez no tan amplio que no nos permita pesquisar la problemática en cuestión" (Erausquin \& D’Arcangelo, 2013, pág. 4).
Por otra parte se plantea la teoría de la actividad según Engestron (como se citó en Baquero, 2002) como unidad de análisis priorizando la actividad colectiva en su conjunto la cual está compuesta por algunos elementos como las reglas y los roles asignados a los diferentes actores implicados así como la comunidad y los instrumentos de mediación superando la visión clásica que se reduce a la relación sujeto - objeto.

Una de las características de la teoría de la actividad manifiesta que esta nos "permite recuperar la dimensión histórica $-\mathrm{y}$ por lo tanto construida- de las prácticas". (Erausquin \& D’Arcangelo, 2013, pág. 11) Sin embargo en lo que compete a nuestra realidad parece necesario construir un instrumento de análisis que recoja y permita entre ver con mayor precisión en la cotidianeidad escolar los desarrollos teóricos que hasta aquí se han planteado frente a las unidades de análisis.

Esto encuentra fundamento también en lo que Dewey) (Como se citó en Rogoff, 1997) refiere al entorno social y es que éste

“...es verdaderamente educativo en sus fines en la medida en que el individuo comparta o participe en algún tipo de actividad conjunta, participando en una actividad asociativa, el individuo se apropia del propósito que la provoca, se familiariza con sus métodos y temas, adquiere la habilidad necesaria, y se impregna de su espíritu emocional". (pág. 2)

Siguiendo la línea de análisis hasta aquí propuesta de la unidad de análisis orientada hacía un recorte consciente de los elementos contextuales que permita una visión amplia de la situación manteniendo las características de conjunto aun en el recorte me permito presentar un esquema al que he denominado Circulo Bio-Geo-Gráfico con el fin de 1) Proponerlo como unidad de análisis, 2) Proponerlo como cartografía o herramienta de lectura-zoom- que guíe la unidad de análisis 3) Proponerlo como parte de los legajos escolares. 


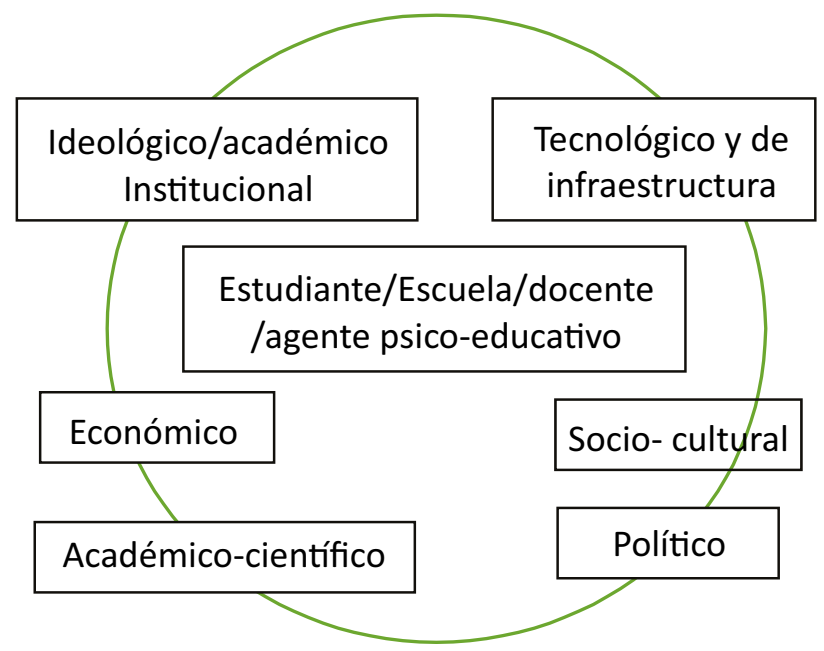

En el centro del Circulo Bio-Geo-Gráfico se ubicará el actor a caracterizar el cual puede ser 1) la escuela, 2) el estudiante 3) el docente, y por qué no 4) el agente psico-educativo, en este sentido podremos observar como confluyen e interactúan las características de estos cuatro actores que si bien se muestran en cuadros individuales llegan al punto de ser como una colonia de hormigas en donde su accionar principalmente dentro de la comunidad educativa es indiferentemente individual ya que este tendrá repercusión en los otros así como los otros la tienen en él.

La intención primaria es poder visualizar posibles discrepancias y posibles puntos de convergencia entre los actores discerniendo la capacidad de adaptación que cada uno tiene frente a la diferencia del otro. Dicho de esta manera se podría plantear o indagar más a fondo por ejemplo el posible conflicto que hay entre las políticas a nivel gubernamental, lo cultural del país o región y el carácter tradicional heredado de la modernidad que aún guardan algunas escuelas y que si bien no es en su totalidad se puede convenir que aún quedan restos sobre todo en lo ideológico y en los métodos de enseñanza. En contraposición a esto el panorama actual en donde las nuevas subjetividades de los estudiantes chocan con un modelo de educación que en su transición hacia un nuevo tipo de escuela no ha encontrado -en algunos casos- concordancia con cuestiones como éstas bien sea desde sus normas o desde sus prácticas educativas.
En este sentido se le apuntaría a la convergencia desde los lugares de vecindad que componen cada uno de los círculos o actores círculos de los actores- en un territorio común sin desprenderse de sus características. En el caso del gráfico estaría detallado en el punto $\mathrm{X}$ el lugar de convergencia sin abandonar su propio lugar de enunciación.

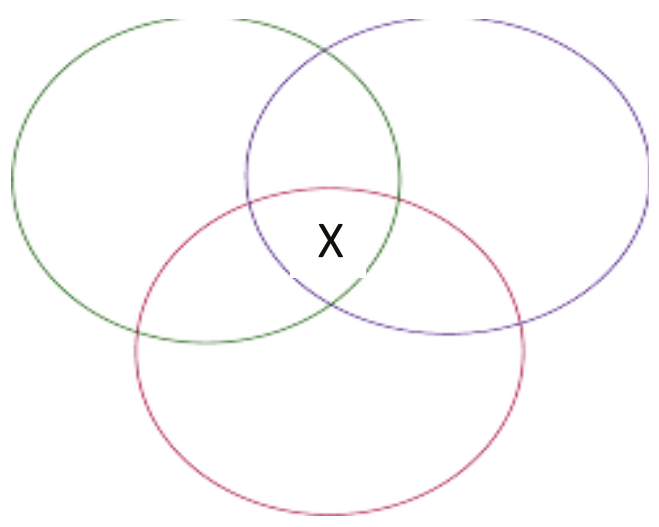

La propuesta del Circulo Bio-Geo-Gráfico responde a la línea propuesta por Vygotsky que Sulle (2014) recupera, como el aporte de herramientas teóricas para pensar el problema de la relación del hombre, la cultura y la sociedad, que permitieron entender que el desarrollo cognitivo es intrínsecamente un proceso social, cultural e histórico y ha contribuido con unidades de análisis y categorías, generadoras de nuevas miradas para comprender y explicar los procesos de desarrollo psicológico y de aprendizaje en contextos cotidianos y escolares. (pág.)

Dicho contexto o análisis contextual al que se aproxima el Circulo Bio-Geo-Gráfico se lo determina "como un factor inherente, ya que no constituye una variable externa o incidente, sino inherente, por lo tanto inseparable de los procesos de aprendizaje y desarrollo. Rodrigo (como se citó en Baquero y Limón, 2001).

De igual manera la propuesta del círculo encuentra coherencia en los planteamientos de Rodrigo (1997) en donde desarrolla la idea del constructivismo diferencial frente al constructivismo unitario. En este sentido descarta la idea de que "...el constructivismo quede caracterizado como un proceso unitario, aplicable a cualquier tipo de conocimiento" 
(Pág. 2), sino más bien propone la idea de constructivismo diferencial la cual se enraíza en la idea de reconocer "la epistemología que guía y da sentido a los tres tipos de conocimiento ${ }^{1}$, ya que de ningún modo debemos suponer que sea la misma". (Rodrigo, 1997, pág. 2). Por lo tanto el círculo nos permitirá en cierta medida discernir las características de los actores en el campo educativo refiriéndome a estos actores tanto al estudiante, docente y agente psico-educativo como a la escuela misma.

A esto Rodrigo (1997) agrega que “... el constructivismo diferencial tiene también en cuenta el escenario de la construcción... de conocimiento" (pág. 2) y complementa con la idea de que "no podemos quedarnos sin más con la caricatura de que el escenario del conocimiento cotidiano es eminentemente social y afectivo, mientras que el escenario científico es un contexto de descubrimiento y el escolar es un contexto instruccional". (Rodrigo, 1997, pág. 2). Por lo que pensar estas características de manera aislada condenaría a las prácticas e intervenciones psico-educativas a un posible fracaso orientando una solución que responda al momento y no a un proyecto de transformación que consolide el trabajo de la psicología en el campo educacional.

De igual manera para Coll (1995) "no existe... ninguna razón de peso para no incluir en la psicología de la educación el estudio de los procesos de cambio comportamental provocados o inducidos por prácticas educativas no escolares" (pág.13) En este línea la mirada complementaría que nos permite el Circulo Bio-Geo-Gráfico nos da más elementos a la hora de interpretar una situación en donde se vean involucrados dos o más sujetos -de por si diferentes- en un contexto determinado aunque procedentes de contextos determinados.

Históricamentesehapensadoque“lapsicología de la educación responde al convencimiento de que la aplicación coherente de los principios psicológicos puede ser altamente beneficiosa para la educación y la enseñanza" (Coll, 1995, pág. 12). De hecho si nos fijamos en "...un pasado no muy lejano bien cabe afirmar que la psicología ha contribuido significativamente para quitar a la escuela su carácter carcelariorepresivo y convertirla en un centro mucho más grato y eficaz del desarrollo humano." (MartínBaró, 2002, pág 47.) Sin embargo nada está dicho y la realidad nos muestra "...la necesidad de que la psicología de la educación tenga en cuenta las características peculiares de las situaciones educativas en la elaboración de sus explicaciones y propuestas..." (Coll, 1995, pág. 12).

El trabajo de la psicología en la educación se presenta como un gran reto ya que la escuela actual "presume que todos los sujetos transitan un mismo proceso de desarrollo. Por lo que las obvias y a veces arduas diferencias entre sujetos quedan signadas como niveles relativos de desarrollo o desvíos o aproximaciones a la norma" (Baquero, 2002, pág.4). Sumado a esto tenemos que al hacer foco en una situación particular se corre el riesgo de obviar el "marco institucional en el que tienen lugar los fenómenos educativos analizados -escuela, familia, centro de formación profesional, empresa...-, de la edad de los sujetos -niños, adolescentes, adultos...y de la naturaleza del contenido -hechos, conceptos, explicaciones, destrezas, actitudes, normas de comportamiento, valores..." Piolat (como se citó en Coll, 1995).

Indudablemente uno de los problemas que aqueja a las prácticas educativas en la escuela es que "...el énfasis contemporáneo en la información, en estar informados, y toda la retórica destinada a constituirnos como sujetos informantes e informados, no hace otra cosa que cancelar nuestras posibilidades de experiencia" (Larroza, 2003, pág. 3).

Esto se debe a que no se reconoce aun que "el conocimiento cotidiano del alumno es particularmente resistente al cambio y no responde adecuadamente a la presentación de contradicciones y discrepancias por parte

1 Se refiere al del hombre de la calle, al científico y al alumno. En "El hombre de la calle, el científico y el alumno: ¿Un solo constructivismo o tres?, en Novedades educativas $\mathrm{N}^{\circ} 76,59-61$. 
del profesor" (Larroza, 2003, pág. 7). En otras palabras "el proceso de escolarización parece fomentar la idea de que "su juego" es aprender reglas simbólicas de diferentes clases, sin que se suponga que haya continuidad entre lo que uno conoce fuera de la escuela y lo que uno aprende en ella". Engestrom) (como se citó en Erausquin y D'Arcangelo, 2013).

$\mathrm{Al}$ parecer la experiencia educativa en su tradición moderna de la escuela como centro de transmisión/repetición/información de conocimiento necesita urgentemente mutar hacía un proceso escolar en donde la realidad no esté desvinculada de lo que se vivencia en el aula y en la institución educativa en general ya que "hay evidencia creciente de que no sólo la escolarización puede no contribuir de un modo directo al desempeño fuera de la escuela, sino además que ese conocimiento adquirido fuera de la escuela no es usado siempre para apoyar el aprendizaje escolar." Engestrom) (como se citó en Erausquin y D'Arcangelo, 2013). En este sentido podemos concluir que las experiencias escolares están siendo separadas de lo cotidiano por lo que no estaría de más echar un vistazo al mundo del estudiante o del docente fuera de la escuela así como en el contexto actual e histórico en el que se constituyó esta institución y en el cual actualmente reside.

\section{El Circulo Bio-Geo-Gráfico como parte del legajo escolar ${ }^{2}$}

Si bien este documento consigna información sobre el estudiante se reduce a la mirada del docente con respecto al comportamiento del chico dentro del aula de clase. En este sentido dicho registro se convierte en una especie de diario de campo donde se sistematizan o registran las estrategias de intervención llevadas a cabo dentro del aula ya sea por los mismos docentes o por los agentes psico-educativos. Los textos de los legajos son "...la reunión de elementos comprobatorios de un proceso de intervención y fundamentalmente de un proceso de evaluación diagnóstica de los alumnos (Toscano, 2007, pág. 4).

"La obra se bautiza con el nombre del personaje al que refieren todos sus informes: el alumno" (Toscano, 2007, pág. 4), y el círculo Bio-GeoGráfico permitirá ampliar la visión de lo que pasa sólo en la escuela trayendo una parte de la realidad que el estudiante vivencia en su día a día fuera de la escuela.

Uno de las instancias en las que los legajos juegan un papel fundamental, es decir, en donde según Toscano (2007) se convierten en la referencia objetiva del proceso es al momento de pensar en la derivación del estudiante a otro tipo de escuela. Sin embargo durante este proceso "los informes y registros que contiene se trasladan de una dependencia a otra del sistema, sobre ellos se negocian criterios, se establecen las discrepancias y se firman acuerdos de lo que se considera la mejor opción para el alumno" (Toscano, 2007, Pág. 5).

Si la decisión va a ser tomada por las diferentes instancias administrativas sería bueno que se tuviese en cuenta la propuesta del círculo Bio-Geo-Gráfico pues es un eco de la voz del estudiante durante el proceso ya que según Toscano (2007) “...el destino escolar del niño lo definen los inspectores en base al análisis de la información recogida a lo largo de los años, delimitada y ordenada en el legajo. (pág. 9). En este sentido si consideramos “...que la decisión final del caso será tomada por una persona ajena al proceso y considerando los elementos de análisis que el texto del legajo le provea" (Toscano, 2007, Pág. 9), sumar al legajo la propuesta del círculo sería darle más información sobre el caso para orientar aún mejor la decisión que implicará la continuación de la trayectoria escolar del estudiante en cuestión.

2 En Argentina los legajos escolares son documentos que contienen información confidencial sobre el alumno en forma de un conjunto heterogéneo de registros e informes. Su apertura refiere generalmente a problemas que presenta en su paso por la escuela. En ellos es posible leer la historia de los procedimientos e intervenciones que los profesionales y docentes de la institución ponen en marcha para evaluar e intervenir sobre las dificultades (Toscano, 2007). 
No es un dato menor recordar que "cuando la derivación a una escuela especial o una estrategia de integración se define, se prepara el legajo para ser presentado ante las inspectoras del distrito quienes avalan o rechazan el pedido" (Toscano, 2007, Pág. 8).

Para Toscano (2007) resulta paradójico que este tipo de decisiones que deciden el destino escolar de los estudiantes tengan que ser tomadas por alguien que no ha llegado a conocerlo o a simplemente tener algún tipo de contacto con él razón por la cual tener consignada la información que proporciona el círculo dentro del legajo puede que de nuevos argumentos para tomar tan significativa decisión.

\section{Conclusiones}

El Circulo Bio-Geo-Gráfico no pretende ser la solución definitiva o el instrumento universal que lleve a hacer el recorte perfecto de la situación, pretende ser un apoyo o complemento al proceso de lectura que hacen los agentes psico-educativos de las diversas situaciones que demandan un proceso de intervención.

Si bien presenta una estructura ésta puede ser modificada según se vaya haciendo necesario pues se entiende que cada sujeto, cada escuela, cada situación es única y particular y que círculo es una herramienta más al momento de realizar un diagnóstico psico-educativo.

Es la posibilidad de complementar la información que las autoridades de la escuela tienen sobre los estudiantes por medio de los legajos favoreciendo la toma de decisiones sobre el futuro escolar de los mismos.

\section{Referencias}

Baquero, R. (2002) “Del experimento escolar a la experiencia educativa. La transmisión educativa desde una perspectiva psicológica situacional" En perfiles educativos. Tercera época. Vol. XXIV. Nos 97-98. Pp. 57-75. México (Fragmentos articuladores).
Baquero, R. (2012) Vigotsky: sujeto y situación, claves de un programa psicológico. Carretero, M. \& Castorina, J. (comps) Desarrollo cognitivo y educación I. Los inicios del conocimiento (61-86). Buenos Aíres: Paidós.

Coll, C. (1995) "Pisología y educación: aproximación a los objetivos y contenidos de la psicología de la educación" en Desarrollo psicológico y educación. Madrid: Alianza editorial.

Erausquin, C. y D'Arcangelo, M. (2013) "Unidades de análisis para la construcción de conocimientos e intervenciones en escenarios educativos". Ficha de cátedra psicología educacional. Publicaciones CEP Facultad de Psicología UNLP y UBA

Larroza, J. (2003) "Experiencia y pasión". En la experiencia de la lectura. Estudios sobre literatura y formación. México. FCE.

Martín-Baró, I. (2002) Psicología de la liberación para América Latina (1942/1989). Pacheco, G; Jiménez, B. (Comps.) ITESO.

Rogoff, B. (1997) "Los tres planos de la actividad sociocultural: aproximación participativa, participación guiada y aprendizaje", en: Wertsch, Del Rio Y Álvarez A. (Eds.) La mente sociocultural. Aproximaciones teóricas y aplicadas, Madrid, Fundación infancia y aprendizaje

Sulle, A. (2014) "Los enfoques socio-culturales. Unidades de análisis y categorías que interpelan las relaciones entre aprendizaje escolar, contex to e intervención psicoeducativa". Ficha de cátedra. Publicaciones CEP. Facultad de psicología UBA.

Toscano, A (2007) "La construcción de Legajos Escolares: Una decisión en torno al destino escolar de los niños". Ficha Cátedra CEP.

Vygotsky, L. (1995). Cap. 1. "El problema y el método de investigación". En Pensamiento y lenguaje (pp. 49-56). Barcelona: Paidós. 